\title{
HERPETOFAUNA DEL PARQUE NACIONAL DE MONTE ALÉN (RÍO MUNI, GUINEA ECUATORIAL)
}

\author{
C. A. Lasso *, A. I. Rial *, J. Castroviejo ** e I. De la Riva ***(1)
}

\begin{abstract}
RESUMEN
En el marco de un estudio general sobre de la diversidad de vertebrados del Parque Nacional de Monte Alén (Río Muni, Guinea Ecuatorial), se identificaron 16 familias, 47 géneros y 65 especies de reptiles, y 7 familias, 29 géneros y 56 especies de anfibios; 21 especies de reptiles y 4 de anfibios representan nuevos registros para la región de Río Muni. En el presente trabajo se expone el inventario preliminar de especies e información ecológica puntual sobre las mismas. Monte Alén puede ser considerada una de las áreas protegidas de mayor diversidad herpetológica de África central.
\end{abstract}

Palabras clave: África, Anfibios, Diversidad, Guinea Ecuatorial, Reptiles, Río Muni.

\begin{abstract}
Herpetofauna of Monte Alén National Park (Rio Muni, Equatorial Guinea)

In the framework of a broad study of the vertebrate diversity at Monte Alen National Park (Río Muni, Equatorial Guinea), we identified 16 families, 47 genera, and 65 species of reptiles and seven families, 29 genera, and 56 species of amphibians. 21 species of reptiles and four species of amphibians represent new records for the Río Muni region. We present the preliminary inventory of the herpetofauna as well as some ecological comments. Monte Alén is considered one of the most diverse protected areas in central Africa from a herpetological standpoint.
\end{abstract}

Key words: Africa, Amphibia, Diversity, Equatorial Guinea, Reptiles, Río Muni.

\section{Introducción}

Dentro de la reducida extensión territorial de Guinea Ecuatorial (aproximadamente $28.000 \mathrm{~km}^{2}$ ) y del notable desconocimiento de su fauna, la región de Monte Alén, situada en la zona centro-occidental de Río Muni (zona continental del país y la de mayor extensión, con $26.000 \mathrm{~km}^{2}$ ), se identificó hace tiempo como merecedora de conservación prioritaria por su riqueza biológica y buen estado de preservación (Castroviejo et al., 1986), lo que llevó en 1988 a la declaración de Monte Alén como área protegida. Posteriormente, el Programa "Conservación y Utilización Racional de los Ecosistemas Forestales de Africa Central" (Proyecto ECOFACComponente Guinea Ecuatorial), puso en marcha durante los años 1993-1995 el estudio de los vertebrados del ya denominado Parque Nacional de

\footnotetext{
* Fundación La Salle, Museo de Historia Natural La Salle, Aptdo. 1930, Caracas 1010 - A, Venezuela

** Asociación Amigos de Doñana, C/ Panamá 6, 41012 Sevilla, España.

*** Museo Nacional de Ciencias Naturales, CSIC, C/ José Gutiérrez Abascal 2, 28006 Madrid, España

(1) Autor para correspondencia.
} 
Monte Alén (en adelante, PNMA) con el objetivo de elaborar un inventario de especies y conocer su distribución en los diferentes hábitats. Dicho estudio puso de manifiesto la elevada biodiversidad del PNMA, que cuenta al menos con 526 especies de vertebrados (De la Riva, 1994a; Lasso, 1995). A esta alta diversidad contribuye el hecho de que la región está constituida por bosques tropicales de la cuenca del Congo, dominados por "pluvisilva densa ecuatorial de mediana y baja altitud" (Keay, 1959), la cual constituyó un área con abundantes refugios forestales pleistocénicos (Moreau, 1963, 1966; Carcasson, 1964; Hamilton, 1976; Maley, 1987, 1991).

Con la excepción de los anfibios anuros, estudiados por De la Riva (1994b), en general la herpetofauna del PNMA constituye el grupo de vertebrados menos estudiado no sólo

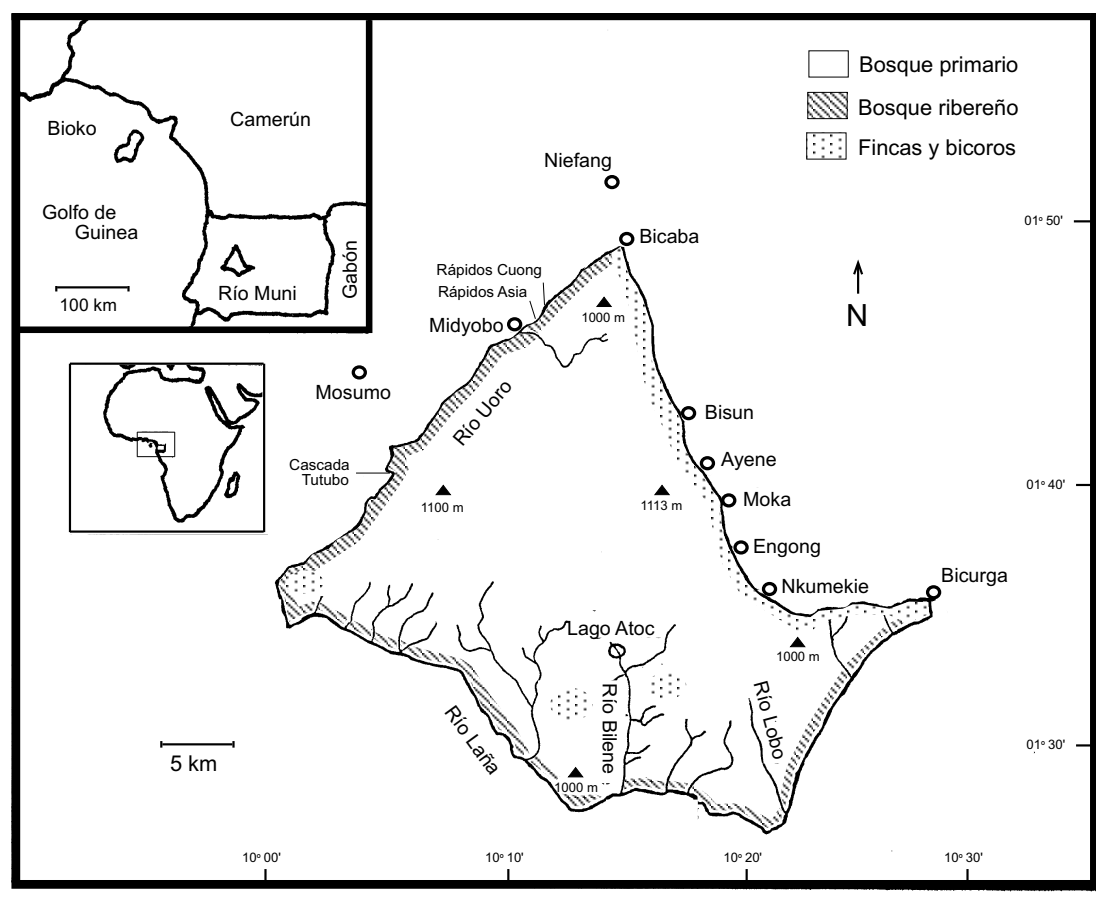

Fig. 1.- Área de estudio. Los límites del Parque son los que tenía cuando se realizó el trabajo, extendiéndose en la actualidad notablemente hacia el suroeste.

Fig. 1. - Study area. The Park boundaries are the ones it had when the study was carried out; currently, the limits has been greatly expanded to the southwest.

del Parque, sino incluso de Río

Muni. Desde el siglo XIX hasta inicios del XX, Boulenger (1899, 1900, 1903, 1906), describió o citó 27 especies válidas de anfibios para Río Muni. En 1993, De la Riva, prospectó el PNMA e indicó la presencia de 49 especies de anfibios anuros, 24 de las cuales no habían sido citadas previamente en Río Muni (De la Riva, 1994b). Dos recopilaciones de datos publicadas recientemente (Blanc y Frétey, 2000; Frétey y Blanc, 2000) arrojan la cifra de 74 especies de anfibios en Guinea Ecuatorial (65 de ellas en Río Muni), lo que sitúa al país, pese a su pequeña extensión, como el tercero más diverso en anfibios de Africa central.

Respecto a los reptiles, Boulenger (1905) mencionó 24 especies en Río Muni, y muy poco se ha publicado desde entonces. Una notable excepción son los trabajos de Knoepffler $(1965,1968)$ sobre los reptiles de la cuenca del río Ntem (= río Campo) y su clave para la identificación de las serpientes de Gabón y áreas limítrofes, Río Muni incluída (Knoepffler, 1974). No obstante, la ausencia general de estudios taxonómicos sobre la herpetofauna ecuatoguineana se refleja en la falta de información referente a este país en las compilaciones de la

UICN (1990) y en trabajos generales como, por ejemplo, los de Hughes (1983), Meirte (1992) y Chippaux (1999) sobre las serpientes de Africa. Más recientemente, Blanc y Frétey (2002) dan la cifra de 136 especies de reptiles en Guinea Ecuatorial, subrayando de nuevo la enorme riqueza que este número representa para un país de tan reducida extensión.

Dado el notable desconocimiento y el vacío de datos existente, en este trabajo nos planteamos el objetivo de proporcionar un inventario preliminar de las especies de anfibios y reptiles del PNMA, aportando información puntual sobre la distribución y ecología de algunas de ellas en el Parque.

\section{Área de estudio}

El PNMA se sitúa en la región continental de Guinea Ecuatorial (Río Muni) y pertenece a la Provincia Centro-Sur, Distrito de Niefang. En la época del estudio, el Parque ocupaba una superficie triangular de $800 \mathrm{~km}^{2}$ en la franja latitudinal entre 


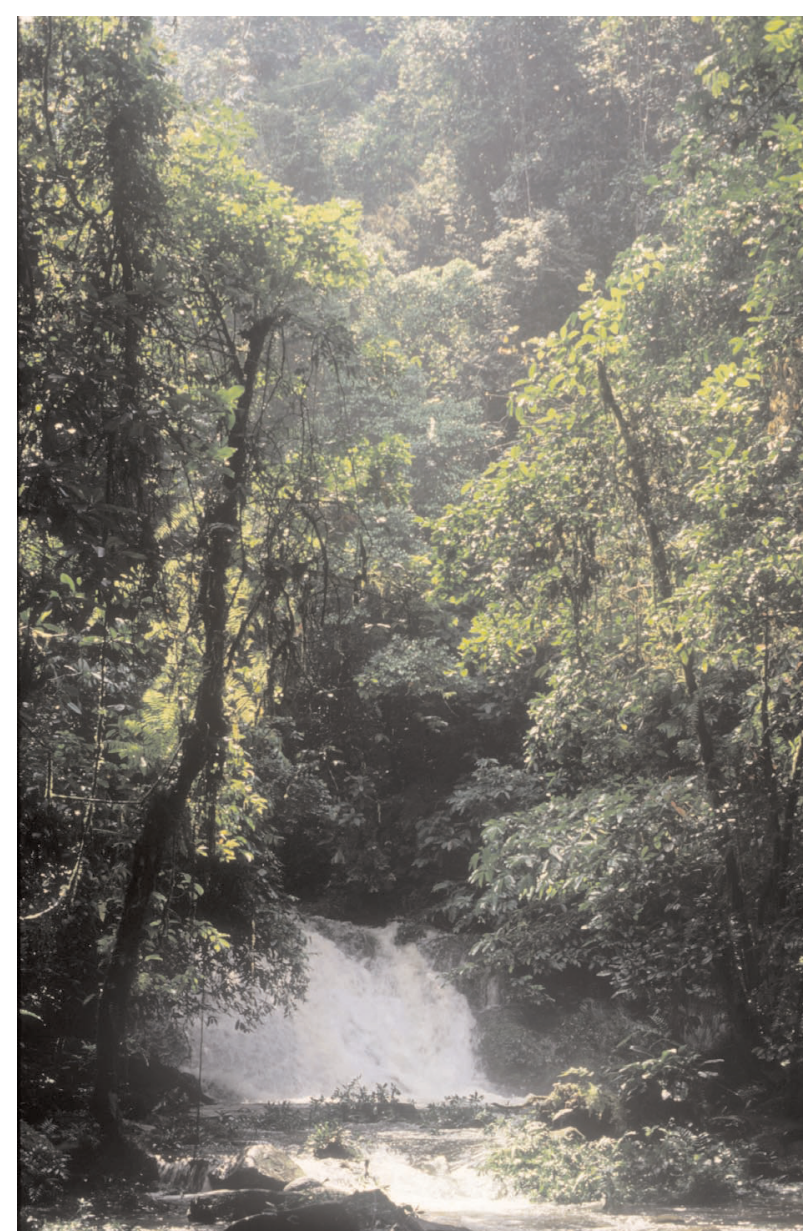

Fig. 2.- Río en bosque primario en el P. N. Monte Alén.

Fig. 2.- River in primary forest at Monte Alén National Park.

los $01^{\circ} 27^{\prime} \mathrm{N}-01^{\circ} 48^{\prime} \mathrm{N}$; posteriores ampliaciones de sus límites hacia el suroeste en 1997 y 2000 han llevado al Parque a su extensión actual de $2000 \mathrm{~km}^{2}$. La porción del PNMA que fue objeto de este estudio se asienta enteramente en la Cadena Montañosa de Niefang, al sur de la Sierra de Mayale y en la Cuenca del Rio Uoro, siendo sus límites los ríos Uoro y Laña y la carretera Niefang-Evinayong (Fig. 1). Los suelos de la zona proceden de la descomposición de la roca madre (gneis y granito) y sufren constantes procesos de laterización ( $\mathrm{Fa}$, 1991). El paisaje es montañoso y presenta cimas en torno a $1000 \mathrm{~m}$ de altitud.

El clima de la región es cálido-húmedo, con patrones de precipitación que definen dos estaciones húmedas y dos secas. La precipitación total anual es del orden de 2000-3000 mm. Según

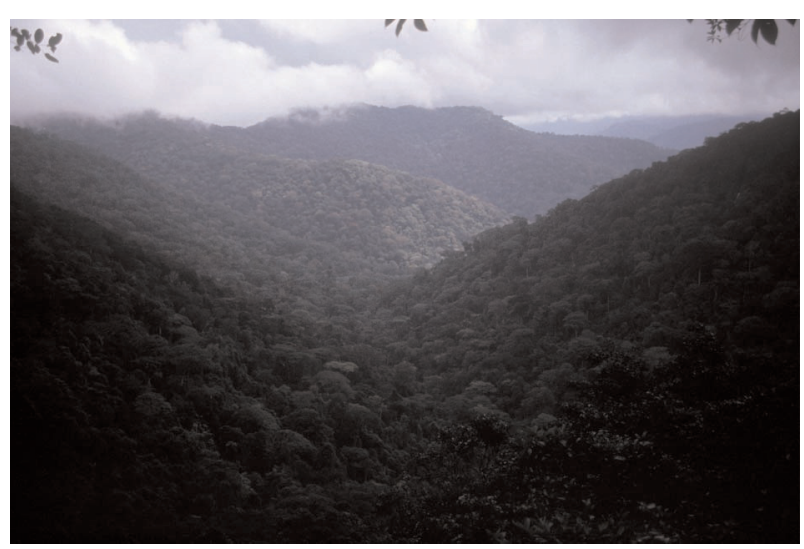

Fig. 3.- Dosel del bosque primario del P. N. Monte Alén desde el mirador de Moka.

Fig. 3.- Canopy of the primary forest at Monte Alén National Park from a wiewpoint near Moka.

Sabater Pi (1984), en la zona de Monte Alén la temperatura media es de $20.5^{\circ} \mathrm{C}$, variando entre $19.5^{\circ} \mathrm{C}$ y $21.9^{\circ} \mathrm{C}$, siendo la mínima $17.2^{\circ} \mathrm{C}$ y la máxima $25.0^{\circ} \mathrm{C}$. Los hábitats acuáticos son en su mayoría lóticos, y lo constituyen ríos y arroyos de aguas cristalinas (Fig. 2) ligeramente ácidas ( $\mathrm{pH} 5,9-6,9)$, de escasa conductividad (15,5-28,9 Ms/cm), muy oxigenadas (4,2-8 ppm) y con temperaturas medias de $23^{\circ} \mathrm{C}$ (Lasso, 1995). Las aguas estancadas, pozas y charcas temporales son escasas dada la orografía del terreno. El único ambiente léntico permanente de cierta importancia en el Parque lo constituye el Lago Atoc, el cual no obstante, simplemente representa una zona de desborde del río Bilene.

Los hábitats terrestres del Parque han sido clasificados por Guinea (1946), Sabater Pi (1984) y Juste (1993), siendo posible reconocer dos tipos básicos de formaciones vegetales:

- Formaciones secundarias y terciarias; son bosques transformados por acción humana, tales como bicoros o bosques secundarios, y formaciones terciarias o heliófilas que colonizan las fincas abandonadas.

- Formaciones primarias o pluvisilva tropical, bosque virgen (Fig. 3). Comprende también los bosques ribereños que crecen junto a los grandes ríos.

Las fincas son pequeñas extensiones agrícolas para el autoconsumo, con plantaciones de diversos productos (yuca, piña, plátano, etc). Las etapas de sucesión que siguen inmediatamente a estos cultivos están dominadas por Aframomum sp. y/o 


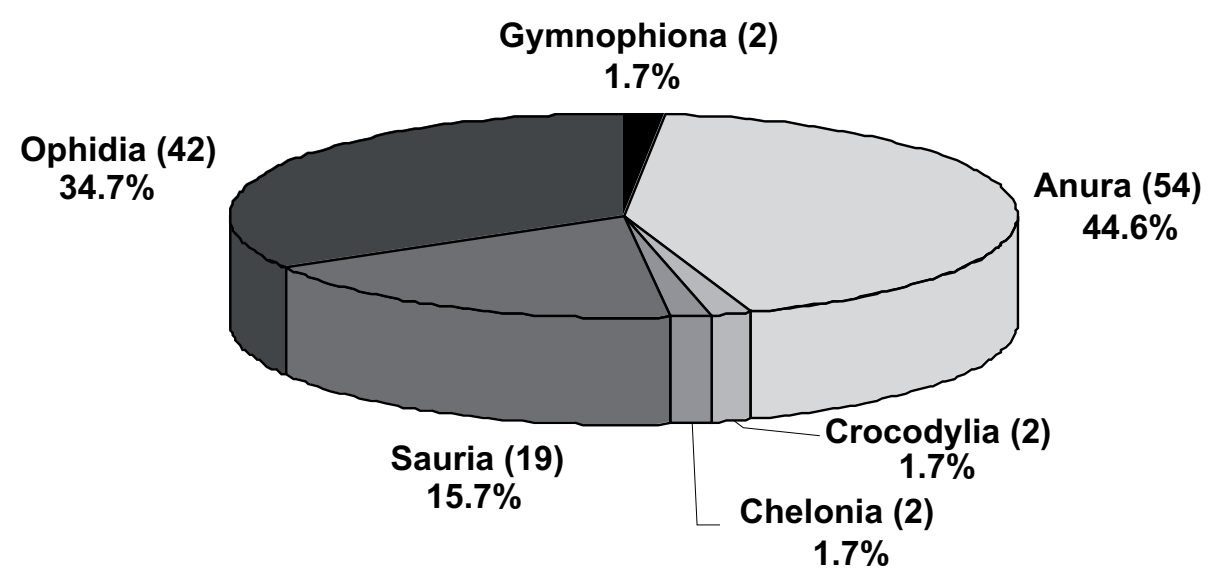

Fig. 4.- Contribución relativa de cada grupo de anfibios y reptiles a la herpetofauna de Monte Alén.

Fig. 4.- Comparative contributions of each main group of amphibians and reptiles to the herpetofauna of Monte Alén.

Musanga cecropioides, mientras que los bicoros estan dominados por árboles, palmas, lianas y epífitas; entre otras especies, predominan Aucomea klaineana, Pycnanthus angolensis, Terminalia superba, Oncocalamus manii, Cissus sp. y Polypodium sp.; los bosques primarios presentan una alta diversidad de especies forestales (Lejoly, 1994), representando fundamentalmente a las familias Caesalpinaceae, Mimosaceae, Moraceae, Sapotaceae, Malvaceae y Burseraceae, con un dosel de 30-35 m de altura y árboles emergentes que superan los $50 \mathrm{~m}$. Las cimas de las montañas pueden constituir los denominados por Guinea (1946) cerros cúpula o "Inselbergs", formaciones de roca madre descubierta, con escaso suelo encharcado, y ocupadas por gramíneas, ciperáceas y orquídeas (ver Parmentier, 1999; Parmentier y Nguema, 2001).

\section{Material y métodos}

Se realizaron prospecciones diurnas y nocturnas desde mayo a diciembre de 1993 (ver De la Riva, 1994b) y desde mayo de 1994 hasta enero de 1995 (Lasso, 1995), cubriendo los diferentes tipos de hábitat del Parque. Los ejemplares recolectados para la colección de referencia se capturaron a mano y con trampas tipo "pitfall" para las especies del sotobosque, y fueron fijados en formol al 10\% y posteriormente conservados en etanol al $70 \%$. En el laboratorio las especies fueron identificadas mediante comparación con las colecciones de referencia del Centro de Estudios Tropicales de Sevilla (CET) (donde se depositó el material), la Estación Biológica de Doñana (EBD), y el Museo de
Historia Natural de París (MNHN). Para la identificación mediante revisión bibliográfica de cada grupo se utilizaron los trabajos de Angel (1933), Boulenger (1887), Hughes (1983), Knoepffler (1968), Loveridge (1972), Meirte (1992), Perret (1973, 1975), Schmidt (1919) y Villiers (1963) para los reptiles, y de Boulenger $(1899,1900,1903$, 1906), De la Riva (1994b) y Perret (1966) para los anfibios. Los ejemplares de identificación dudosa fueron llevados al Museo de Historia Natural de París para comprobación taxonómica con la ayuda de expertos (ver agradecimientos). La nomenclatura de reptiles sigue fundamentalmente la base de datos de Uetz (2002; EMBL Reptile Database (http://www.reptile-database.org) y, la de anfibios, fundamentalmente la de Frost (2002; http://research.amnh.org/cgi-bin/herpetology/amphibia).

\section{Resultados}

La lista de especies obtenida (Tablas 1 y 2 ) proviene por completo de los datos propios de los autores, parcialmente recopilados y comentados en los trabajos de De la Riva (1994a, 1994b) y en el de Lasso (1995), cuya información global fue ya recogida por Frétey y Blanc (2000) para compilar su lista faunística de los anfibios de Africa central.

La fauna herpetológica de Monte Alén se compone al menos de 121 especies, de las cuales 65 especies (un 53.7\%) son reptiles correspondientes a tres órdenes, 16 familias y 47 géneros, siendo el grupo más diverso el de las serpientes, que, con 42 especies (26 de ellas de la familia Colubridae), representan un $65 \%$ de la diversidad reptiliana. Los anfibios están representados por 56 especies (un 
$46.3 \%$ de la herpetofauna) repartidos en dos órdenes, siete familias y 29 géneros (Fig. 4). La lista de especies de reptiles y su distribución ecológica dentro del Parque se muestra en la Tabla 1, donde las especies con un asterisco representan nuevos registros para Río Muni. La lista de especies de anfibios y su distribución ecológica dentro del Parque se muestra en la Tabla 2, donde las especies con un asterisco al final del nombre representan nuevos registros para Río Muni, y aquellas que lo tienen al principio del nombre son registradas para el Parque por primera vez.

\section{REPTILIA}

\section{CROCODYlia}

Hay, o había, dos especies de cocodrilos en el Parque, ambas incluidas en el Apéndice I del CITES. Osteolaemus tetraspis se distribuye a lo largo de todo el curso del río Laña, especialmente en su parte baja y principales tributarios como los ríos Lobo, Bilene (incluyendo el lago Atoc), Bibas y Mensang. También se encuentra en el río Uoro y afluentes (Bong, Mongó y Ñamienle), pero es mucho más escaso que en el Laña debido principalmente a la cacería furtiva, que incide sobre todo desde Niefang hasta la confluencia del Uoro con el Laña.

La existencia de poblaciones de Crocodylus cataphractus en los ríos Uoro y Laña mencionadas por Fa (1991) requiere confirmación actual. Juste y Cantero (1991) lo consideraron probablemente extinto en Río Muni, y no había una evidencia concluyente de su presencia en 1994 en el PNMA. Sin embargo, los residentes del área, especialmente entre los poblados de Midyobo y Mosumo, lo conocen bien, aunque dicen no haberlo visto desde hace muchos años. Nosotros tuvimos la oportunidad de examinar un cráneo de un ejemplar adulto cazado en 1984 en el río Uoro. El indicio más reciente de su presencia en el Parque, se refiere a unas huellas atribuíbles a esta especie observadas por Jesús Elá (jefe de los ecoguardas) en las playas del río Uoro el 24-7-94 (aprox. 01 37'4”'N/1003'51'”E).

\section{ChelONIA}

En el Parque hay dos especies de quelonios, Kinixys erosa y Pelusios gabonensis (Fig. 5), correspondientes a las familias Testudinidae y Pelomedusidae, respectivamente. La primera especie, terrestre, es relativamente común y está ampliamente distribuida, tanto en el boque primario como en el bicoro. La segunda, acuática, es considerablemente más escasa, encontrándose los adultos en los cauces de los grandes ríos, mientras que

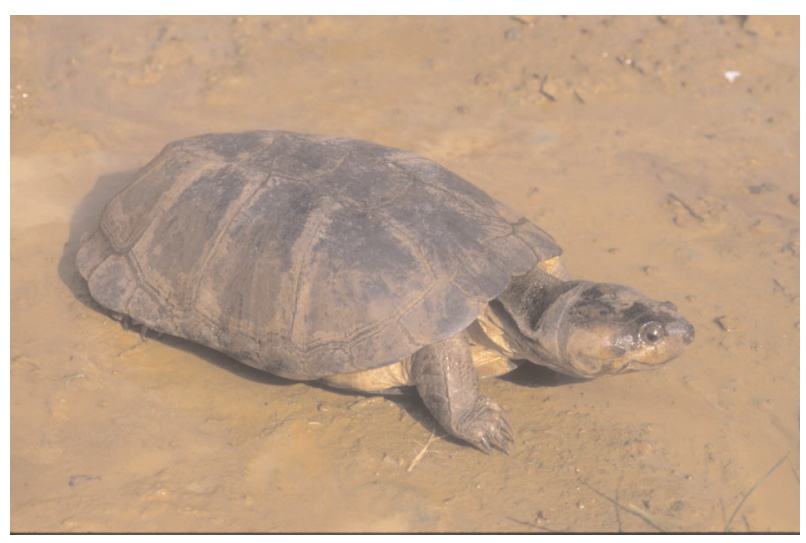

Fig. 5.- Pelusios gabonensis.

los juveniles remontan pequeños afluentes hasta distancias considerables.

\section{SAURIA}

La fauna de saurios del PNMA consta al menos de 19 especies. La familia Lacertidae está representada. hasta ahora, por una sola especie, rara y restringida al bosque primario, Poromera fordi.

Se encuentran tres especies de Gekkonidae, todas del género Hemidactylus. Hemidactylus fasciatus y H. mabouia son comunes en las casas y bicoros, mientras que $H$. muriceus ha sido colectada únicamente en el bosque ripario del río Laña. De las tres especies, sólo $H$. fasciatus había sido ya citada en Río Muni.

La famila Agamidae tiene un solo representante en el Parque, Agama agama, que es el reptil más abundante y común de Monte Alén. Se encuentra en todos los hábitats, con marcada preferencia por los poblados, márgenes de carreteras y bicoros. En el bosque virgen es raro.

Los varánidos están representados por Varanus ornatus, que se encuentra en todo el Parque, especialmente en las zonas bajas aledañas a los ríos Uoro y Laña, donde parece ser más abundante. Se ha observado tanto en bosque primario como secundario e inclusive en fincas agrícolas, pero siempre asociado a cuerpos de agua.

La familia Scincidae está representada por cuatro géneros y ocho especies, de las cuales Mabuya affinis es la más común y ampliamente distribuida. Mochlus fernandi se encuentra en el bosque primario, Panaspis breviceps, $P$. rohdei y $P$. reichenowi pueden habitar tanto en bosques secundarios como en bicoros y Mabuya albilabris y M. polytropis están en todos los hábitats; Feylinia currori aparen- 
Tabla 1.- Lista y distribución ecológica de los reptiles del PNMA. Un asterisco indica que la especie es citada por primera vez para Río Muni. S, suelo; V, vegetación; AC, aguas corrientes; AE, aguas estancadas.

Table 1.- List and ecological distribution of reptiles of Monte Alén National Park. An asterisk indicates that the species is reported in Río Muni for the first time. S, floor; V, vegetation; AC, streams; AE, stagnant water.

\begin{tabular}{|c|c|c|c|c|c|c|c|c|c|c|c|c|}
\hline \multirow[t]{2}{*}{ ESPECIES } & \multicolumn{4}{|c|}{ BOSQUE PRIMARIO } & \multicolumn{4}{|c|}{ BOSQUE SECUNDARIO } & \multicolumn{4}{|c|}{ FINCAS } \\
\hline & $\mathbf{S}$ & $\mathbf{V}$ & $\mathbf{A C}$ & $\mathbf{A E}$ & $\mathbf{S}$ & $\mathbf{V}$ & $\mathrm{AC}$ & $\mathbf{A E}$ & $\mathbf{S}$ & $\mathbf{V}$ & $\mathbf{A C}$ & $\mathbf{A E}$ \\
\hline $\begin{array}{l}\text { CROCODYLIA } \\
\text { CROCODYLIDAE } \\
\text { Crocodylus cataphractus } \text { Cuvier } 1825 \\
\text { Osteolaemus tetraspis Cope, } 1861\end{array}$ & & & $\begin{array}{l}X \\
X\end{array}$ & $\begin{array}{l}X \\
X\end{array}$ & & & & & & & & \\
\hline $\begin{array}{l}\text { TESTUDINATA } \\
\text { PELOMEDUSIDAE } \\
\text { Pelusios gabonensis (Duméril, 1856) }\end{array}$ & & & $\mathrm{X}$ & $\mathrm{X}$ & & & & & & & & \\
\hline $\begin{array}{l}\text { TESTUDINIDAE } \\
\text { Kinixys erosa (Schweigger, 1814) }\end{array}$ & $\mathrm{X}$ & & & & $\mathrm{X}$ & & & & & & & \\
\hline $\begin{array}{l}\text { SQUAMATA } \\
\text { LACERTIDAE } \\
\text { Poromera fordi (Hallowell, 1857) }\end{array}$ & $\mathrm{X}$ & $\mathrm{X}$ & & & & & & & & & & \\
\hline $\begin{array}{l}\text { GEKKONIDAE } \\
\text { Hemidactylus fasciatus Gray, } 1831 \\
\text { Hemidactylus mabouia (Moreau de Jonnes, 1818)* } \\
\text { Hemidactylus muriceus Peters, } 1870^{*}\end{array}$ & $\mathrm{X}$ & $\mathrm{X}$ & & & $\mathrm{X}$ & $\mathrm{X}$ & & & $\begin{array}{l}X \\
X\end{array}$ & $\begin{array}{l}X \\
X\end{array}$ & & \\
\hline $\begin{array}{l}\text { AGAMIDAE } \\
\text { Agama agama Linnaeus, } 1758\end{array}$ & $\mathrm{X}$ & $\mathrm{X}$ & & & $\mathrm{X}$ & $\mathrm{X}$ & & & $\mathrm{X}$ & $\mathrm{X}$ & & \\
\hline $\begin{array}{l}\text { VARANIDAE } \\
\text { Varanus ornatus (Daudin, 1803) }\end{array}$ & $\mathrm{X}$ & $\mathrm{X}$ & $\mathrm{X}$ & $\mathrm{X}$ & $\mathrm{X}$ & $\mathrm{X}$ & $\mathrm{X}$ & $\mathrm{X}$ & $\mathrm{X}$ & $\mathrm{X}$ & $\mathrm{X}$ & $\mathrm{X}$ \\
\hline $\begin{array}{l}\text { SCINCIDAE } \\
\text { Feylinia currori Gray, 1845 } \\
\text { Mochlus fernandi (Burton, 1836) } \\
\text { Panaspis breviceps (Peters, 1873)* } \\
\text { Panaspis reichenowi (Peters, 1874)* } \\
\text { Panaspis rohdei (Muller, 1910)* } \\
\text { Mabuya affinis (Gray, 1839) } \\
\text { Mabuya albilabris (Hallowell, 1857) } \\
\text { Mabuya polytropis Boulenger, 1903* }\end{array}$ & $\begin{array}{l}X \\
X \\
X\end{array}$ & $\begin{array}{l}X \\
X \\
X \\
X\end{array}$ & & & $\begin{array}{l}X \\
X \\
X \\
X \\
X \\
X\end{array}$ & $\begin{array}{l}X \\
X \\
X \\
X \\
X \\
X\end{array}$ & & & $\begin{array}{l}X \\
X \\
X \\
X\end{array}$ & $\begin{array}{l}X \\
X \\
X \\
X\end{array}$ & & \\
\hline $\begin{array}{l}\text { CHAMAELEONIDAE } \\
\text { Chamaeleo cristatus Stutchbury, } 1837 \\
\text { Chamaeleo gracilis Hallowell, } 1844 \\
\text { Chamaeleo oweni } \text { Gray, } 1831 \\
\text { Chamaeleo } \text { sp. } \\
\text { Rhampholeon spectrum (Buchholz, 1847) * }\end{array}$ & $\mathrm{X}$ & $\begin{array}{l}X \\
X \\
X \\
X \\
X\end{array}$ & & & $\mathrm{X}$ & $\begin{array}{l}X \\
X \\
X \\
X\end{array}$ & & & & & & \\
\hline $\begin{array}{l}\text { TYPHLOPIDAE } \\
\text { Typhlops angolensis (Bocage, 1866)* } \\
\text { Typhlops congestus (Duméril y Bribon, 1844)* } \\
\text { Rhinotyphlops wittei (Roux-Estéve, 1974)* }\end{array}$ & $\begin{array}{l}X \\
X \\
X\end{array}$ & & & & $\mathrm{X}$ & & & & $\mathrm{X}$ & & & \\
\hline $\begin{array}{l}\text { LEPTOTYPHLOPIDAE } \\
\text { Leptotyphlops perreti Roux - Estéve, 1974* } \\
\text { BOIDAE } \\
\text { Calabaria reinhardti (Schlegel, 1848) } \\
\text { Phyton sebae (Gmelin, 1788) }\end{array}$ & $\begin{array}{l}X \\
X\end{array}$ & $\mathrm{X}$ & & & & & & & & & & \\
\hline $\begin{array}{l}\text { ATRACTASPIDIDAE } \\
\text { Aparallactus modestus (Gunther, 1859)* } \\
\text { Atractaspis boulengeri matschiensis Werner } 1897^{*} \\
\text { Atractaspis reticulata heterochilus } \text { Boulenger, } 1901\end{array}$ & $\begin{array}{l}X \\
X \\
X\end{array}$ & & $\mathrm{X}$ & & $\mathrm{X}$ & & & & & & & \\
\hline $\begin{array}{l}\text { COLUBRIDAE } \\
\text { Boiga pulverulenta (Fisher, 1856) } \\
\text { Bothropthalmus lineatus (Peters, 1863) } \\
\text { Bothrolycus ater (Günter, 1874) } \\
\text { Buhoma depressiceps (Werner, 1897) } \\
\text { Dipsadoboa unicolor Günther 1858* } \\
\text { Gastropyxis smaragdina (Schlegel, 1937) } \\
\text { Goniotophis brussauxi (Mocquard, 1889)* } \\
\text { Grayia ornata (Bocage, 1869) } \\
\text { Grayia smythii Leach, } 1818 \\
\text { Hapsidophrys lineatus } \text { Fisher, 1856 } \\
\text { Hydraethiops melanogaster Günther, 1872* } \\
\text { Lamprophis olivaceus } \text { (Dumeril, 1856) }\end{array}$ & $\begin{array}{l}X \\
X \\
X \\
X \\
X \\
X \\
\\
X \\
X \\
X \\
X\end{array}$ & $\begin{array}{l}X \\
X \\
X\end{array}$ & $\begin{array}{l}X \\
X\end{array}$ & & $\begin{array}{l}X \\
X \\
X \\
X\end{array}$ & $\begin{array}{l}X \\
X \\
X \\
X\end{array}$ & & & $\begin{array}{l}X \\
X \\
X\end{array}$ & $\begin{array}{l}X \\
X \\
X\end{array}$ & & \\
\hline
\end{tabular}




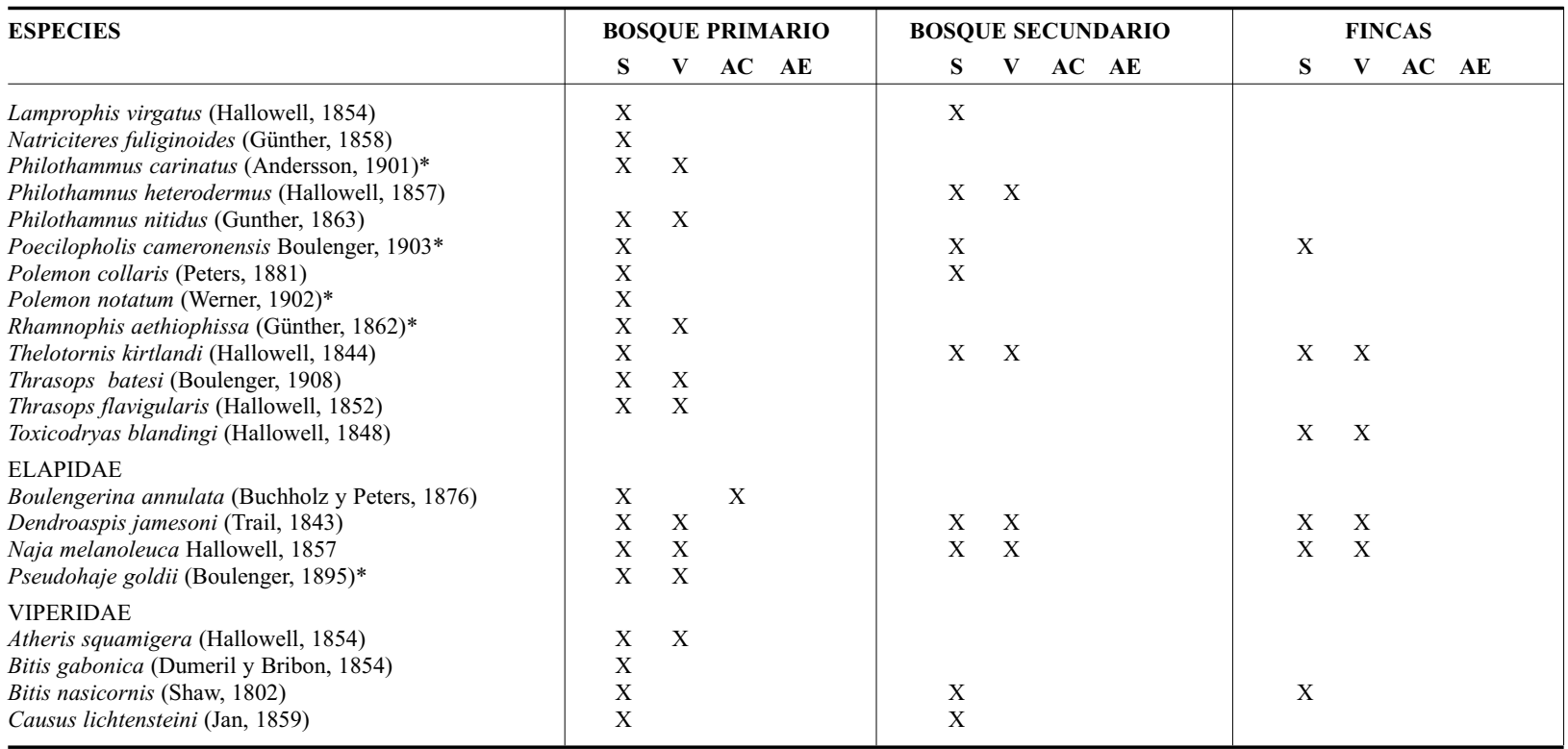

ta ser poco común, ya que se registró tan sólo un ejemplar en una zona de cultivo. Las tres especies de Panaspis y M. polytropis se citan aquí por primera vez para Río Muni, y P. rhodei es primera cita para Guinea Ecuatorial.

Por último, la famila Chamaeleonidae está representada por cinco especies, distribuidas en los bosques primarios y secundarios del Parque. Todas las especies son arborícolas y Rhampholeon spectrum (citado aquí por primera vez para Río Muni; Fig. 6) se encuentra también en el suelo. De las cuatro especies identificadas, Chamaeleo cristatus y $R$. spectrum

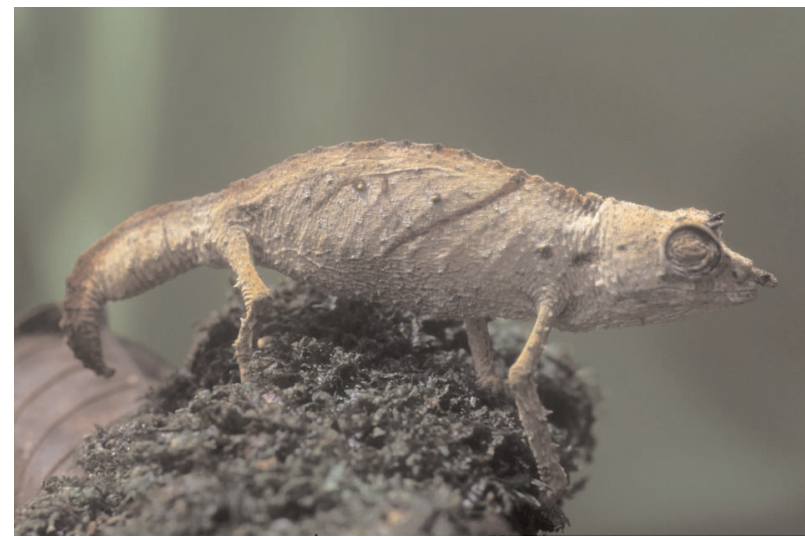

Fig. 6.- Rhampholeon spectrum. son las dos especies más comunes, mientras que $C$. gracilis y $C$. owenii son las más raras. Por último, un solo ejemplar de una especie aún no identificada, aparentemente del grupo cristatus, fue encontrado recientemente (junio de 2002) por miembros de una expedición del Museo Peabody (Universidad de Yale, U.S.A.). (K. Ziskowski, in litt.).

\section{OPHIDIA}

Hasta el momento se han encontrado en el PNMA 42 especies de serpientes, pero esta cifra, con seguridad, no refleja la diversidad real de este grupo en la zona (ver Discusión). Las familias Leptotyphlopidae y Typhlopidae, de hábitos subterráneos, están representadas en Monte Alén por una $\mathrm{y}$ tres especies, respectivamente, y nunca habían sido citadas antes en Río Muni (Typhlops congestus se conoce de Bioko) .

La familia Boidae, está representada por Calabaria reinhardti (Fig. 7) y Phyton sebae. Esta última es muy escasa en las tierras altas y sólo ha sido observada en la confluencia de los ríos Uoro y Laña.

La familia Atractaspididae, serpientes venenosas de hábitos semifosoriales que viven en la hojarasca del bosque, cuenta con tres especies en el Parque, de las que Aparallactus modestus y Atractaspis boulengeri no eran conocidas en Guinea Ecuatorial.

Las 26 especies de colúbridos incluyen especies arborícolas como las de los géneros Boiga, Gastropyxis, Geodipsas, Philothamnus, Rhamnophis, The- 
Tabla 2.- Lista y distribución ecológica de los anfibios del PNMA. Un asterisco al final del nombre indica que la especie es citada por primera vez para Río Muni y al principio del nombre indica que es primer registro para el Parque. Para las especies terrestres se indica el medio acuático en que habitualmente se reproducen.S, suelo; V, vegetación; AC, aguas corrientes; AE, aguas estancadas.

Table 2.- List and ecological distribution of amphibians of Monte Alén National Park. An asterisk at the end of a species name indicates that the species is reported in Río Muni for the first time; an asterisk at the beginning of the name indicates that the species is reported in the Park for the first time. For the terrestrial species, the kinds of aquatic environments where the reproduction takes place are indicated.S, floor; V, vegetation; AC, streams; AE, stagnant water.

\begin{tabular}{|c|c|c|c|c|c|c|c|c|c|c|c|c|}
\hline \multirow[t]{2}{*}{ ESPECIES } & \multicolumn{4}{|c|}{ BOSQUE PRIMARIO } & \multicolumn{4}{|c|}{ BOSQUE SECUNDARIO } & \multicolumn{4}{|c|}{ FINCAS } \\
\hline & $\mathbf{S}$ & $\mathbf{V}$ & $\mathbf{A C}$ & $\mathbf{A E}$ & $\mathbf{S}$ & $\mathbf{V}$ & $\mathbf{A C}$ & $\mathbf{A E}$ & $\mathbf{S}$ & $\mathbf{V}$ & $\mathbf{A C}$ & $\mathbf{A E}$ \\
\hline $\begin{array}{l}\text { GYMNOPHIONA } \\
\text { CAECILIIDAE } \\
\text { *Geotrypetes seraphini (A. Duméril, 1859)* } \\
\text { *Herpele squalostoma (Stutchbury, 1836) }\end{array}$ & $\begin{array}{l}X \\
X\end{array}$ & & $\mathrm{X}$ & & $\begin{array}{l}X \\
X\end{array}$ & & & & & & & \\
\hline $\begin{array}{l}\text { ANURA } \\
\text { ARTHROLEPTIDAE } \\
\text { Arthroleptis adelphus Perret, } 1966 \\
\text { Arthroleptis variabilis } \text { Matschie, } 1893 \\
\text { Arthroleptis sp.1 } \\
\text { Arthroleptis sp.2 } \\
\text { Astylosternus batesi (Boulenger, 1900) } \\
\text { Cardioglossa elegans Boulenger, 1906 } \\
\text { *Cardioglossa escalerae Boulenger, } 1903 \\
\text { Cardioglossa gracilis Boulenger, 1900 } \\
\text { Cardioglossa leucomystax (Boulenger, 1903) } \\
\text { Leptodactylodon albiventris (Boulenger, 1905) } \\
\text { Nyctibates corrugatus } \text { Boulenger, 1904 } \\
\text { Schoutedenella poecilonota } \text { (Peters, 1863) } \\
\text { *Schoutedenella } \text { sp.* } \\
\text { Scotobleps gabonicus } \text { Boulenger, 1900 } \\
\text { Trichobatrachus robustus Boulenger, 1900 }\end{array}$ & $\begin{array}{l}X \\
X \\
X \\
X \\
X \\
X \\
X \\
X \\
X \\
X \\
X \\
X \\
X \\
X \\
X\end{array}$ & & $\begin{array}{l}X \\
X \\
\\
X \\
X\end{array}$ & & $\begin{array}{l}X \\
X\end{array}$ & & $\begin{array}{l}X \\
X\end{array}$ & & $\mathrm{X}$ & & & \\
\hline $\begin{array}{l}\text { BUFONIDAE } \\
\text { Bufo camerunensis Parker, } 1936 \\
\text { Bufo gracilipes Boulenger, } 1899 \\
\text { Bufo latifrons Boulenger, } 1900 \\
\text { Bufo superciliaris Boulenger, } 1887 \\
\text { Bufo tuberosus Günther, } 1958 \\
\text { *Nectophryne afra Buchholz y Peters, } 1875 \\
\text { Werneria mertensiana Amiet, } 1976\end{array}$ & $\begin{array}{l}X \\
X \\
X \\
X \\
X \\
X \\
X\end{array}$ & $\mathrm{X}$ & $\begin{array}{l}X \\
X \\
X \\
X \\
X \\
X \\
X\end{array}$ & $\mathrm{X}$ & $\begin{array}{l}X \\
X \\
\\
X \\
X\end{array}$ & & $\begin{array}{l}X \\
X \\
\\
X\end{array}$ & $\mathrm{X}$ & & & & \\
\hline $\begin{array}{l}\text { HYPEROLIIDAE } \\
\text { Afrixalus brevipalmatus (Ahl, 1931) } \\
\text { Afrixalus paradorsalis Perret, 1960 } \\
\text { Alexteroon obstetricans (Ahl, 1931) } \\
\text { Cryptothylax greshoffii (Schilthuis, 1889) } \\
\text { Hyperolius cinnamomeoventris Bocage, } 1866 \\
\text { Hyperolius ocellatus Günther, } 1858 \\
\text { Hyperolius pardalis Laurent, } 1948 \text { “1947”" } \\
\text { Hyperolius tuberculatus (Mocquard, 1897) } \\
\text { Leptopelis aubryi (Duméril, 1856) } \\
\text { Leptopelis boulengeri (Werner, 1898) } \\
\text { Leptopelis brevirostris (Werner, 1898) } \\
\text { Leptopelis calcaratus (Boulenger, 1906) } \\
\text { *Leptopelis notatus (Buchholz y Peters, 1875)* } \\
\text { Leptopelis ocellatus (Mocquard, 1902) } \\
\text { Leptopelis rufus Reichenow, 1874 } \\
\text { Phlyctimantis leonardi (Boulenger, 1906) }\end{array}$ & & $\begin{array}{l}X \\
X \\
X \\
X \\
X \\
X\end{array}$ & $\mathrm{X}$ & $\begin{array}{l}X \\
X\end{array}$ & & $\begin{array}{l}X \\
X \\
X \\
X\end{array}$ & $\begin{array}{l}X \\
X \\
X \\
X\end{array}$ & $\begin{array}{l}X \\
X\end{array}$ & & $\begin{array}{l}X \\
X \\
X \\
X \\
X\end{array}$ & $\begin{array}{l}X \\
X \\
X \\
X\end{array}$ & $\begin{array}{l}X \\
X\end{array}$ \\
\hline $\begin{array}{l}\text { PIPIDAE } \\
\text { Xenopus fraseri Boulenger, } 1905 \\
\text { *Hymenochirus boettgeri (Tornier, 1897)* }^{*}\end{array}$ & & & $\begin{array}{l}X \\
X\end{array}$ & $\begin{array}{l}X \\
X\end{array}$ & & & $\begin{array}{l}X \\
X\end{array}$ & $\mathrm{X}$ & & & $\begin{array}{l}X \\
X\end{array}$ & $\mathrm{X}$ \\
\hline $\begin{array}{l}\text { RANIDAE } \\
\text { Amnirana albolabris (Hallowell, 1856) } \\
\text { Amnirana amnicola (Perret, 1977) } \\
\text { Amnirana lepus (Andersson, 1903) } \\
\text { Conraua crassipes (Buchholz y Peters, 1875) } \\
\text { Conraua goliath (Boulenger, 1906) } \\
\text { Dimorphognathus africanus (Hallowell, } 1858 \text { “1857”) } \\
\text { Petropedetes newtoni (Bocage, 1895) } \\
\text { Petropedetes palmipes Boulenger, 1905 } \\
\text { Petropedetes parkeri Amiet, 1983 } \\
\text { Phrynobatrachus auritus Boulenger, } 1900 \\
\text { Phrynobatrachus sp. } \\
\text { Phrynodon sandersoni } \text { Parker, 1935 } \\
\text { Ptychadaena aequiplicata (Werner, 1898) }\end{array}$ & $\begin{array}{l}X \\
X \\
X \\
X \\
X \\
X \\
X \\
X \\
X \\
X\end{array}$ & $\begin{array}{l}X \\
X \\
X\end{array}$ & $\begin{array}{l}X \\
X \\
X \\
X \\
X \\
X \\
X \\
X \\
X \\
\\
X \\
X\end{array}$ & $\begin{array}{l}X \\
X \\
X\end{array}$ & $\begin{array}{l}X \\
X \\
X \\
X\end{array}$ & (1) & $\begin{array}{l}X \\
X \\
X \\
X \\
X \\
X \\
X \\
X\end{array}$ & (1) & $\mathrm{X}$ & $\begin{array}{l}X \\
X\end{array}$ & $\begin{array}{l}X \\
X\end{array}$ & $\mathrm{X}$ \\
\hline $\begin{array}{l}\text { RHACOPHORIDAE } \\
\text { Chiromantis rufescens (Günther, } 1869 \text { “1868”) }\end{array}$ & & $\mathrm{X}$ & & $\mathrm{X}$ & & $\mathrm{X}$ & & $\mathrm{X}$ & & $\mathrm{X}$ & & $\mathrm{X}$ \\
\hline
\end{tabular}




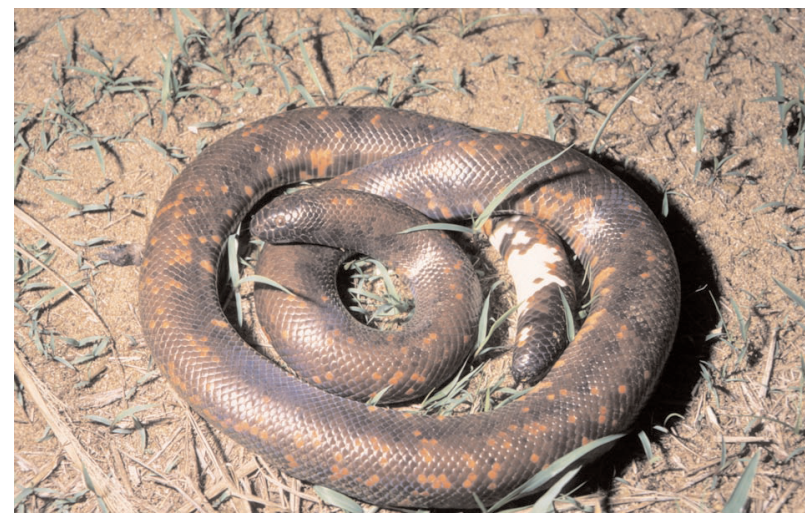

Fig. 7.-Calabaria reinhardti.

lothornis, Thrasops y Toxicodryas; de hábitos semiacuáticos, como las del género Grayia; o acuáticas, como Hydraethiops melanogaster. Se encuentran culebras en todos los hábitats e inclusive algunas especies son muy frecuentes en los asentamientos humanos. Siete especies de colúbridos son citadas aquí por primera vez para Río Muni (Tabla 1).

Las familias Elapidae y Viperidae, con cuatro especies cada una, constituyen el grueso de las serpientes venenosas del Parque. La cobra (Naja melanoleuca) es abundante en todos los hábitats. Pseudohaje goldii se cita aquí por primera vez para Río Muni. Bitis gabonica y B. nasicornis son moderadamente comunes. Durante el período de estudio no se tuvo información de ningún caso de ofidismo.

\section{AMPHIBIA}

Los anfibios del PNMA están incluidos en dos órdenes. El primero, Gymnophiona, agrupa a los anfibios ápodos y está representado en el Parque por la familia Caeciliidae, con dos especies (Geotrypetes seraphini y Herpele squalostoma). La primera especie fue colectada únicamente dentro del agua en ríos de orden mediano, afluentes del Uoro (Michimi y Envoguyi). Herpele squalostoma vive semienterrada en el suelo y, según nuestras observaciones, se muestra activa en superficie inmediatamente después de fuertes lluvias.

El segundo orden, Anura, abarca el grupo más diversificado e incluye hasta el momento 54 especies, correspondientes a 27 géneros y seis familias.

La familia Arthroleptidae está representada por las subfamilias Arthroleptinae (géneros Arthroleptis, Cardioglossa y Schoutedenella, con un total de 10 especies) y Astylosterninae (5 géneros con una especie cada uno). Los Arthroleptinae incluyen for-

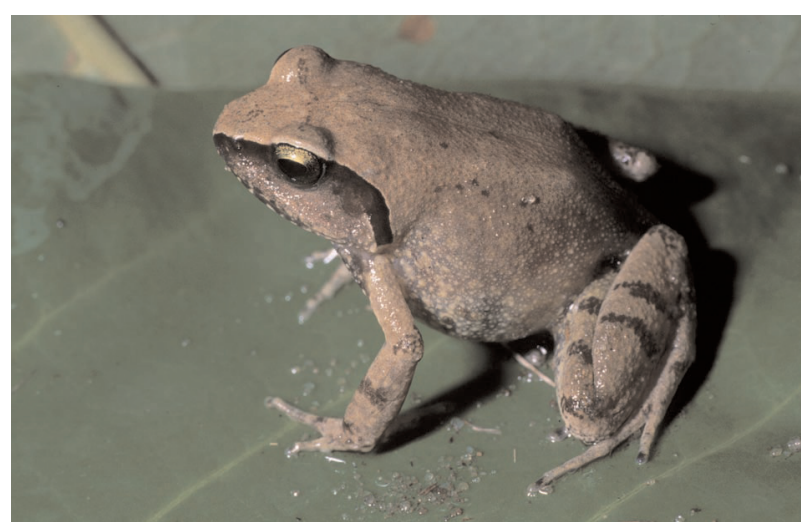

Fig. 8.- Arthroleptis variabilis.

mas típicas del suelo del bosque primario, algunas asociadas a cuerpos de agua. Arthroleptis variabilis (Fig. 8) es una de las especies más comunes y abundantes. Schoutedenella poecilonota es también abundante, hallándose en los tres tipos de hábitat del Parque.

Entre los Astylosterninae destaca Trichobatrachus robustus, especie rara en el Parque y perseguida tanto por su carne como por las creencias populares que la asocian con el vigor que producen en la mujeres embarazadas. Sólo se ha registrado su presencia tres veces, dos en el río Lobo (un macho adulto en celo, con filamentos epidérmicos bien desarrollados, hallado el 25-5-94 y una hembra adulta con huevos hallada el 30-11-94) y una en el río Bilene, junto al lago Atoc (macho adulto sin filamentos desarrollados) (De la Riva, 1994b). El estatus poblacional de esta especie en Río Muni es indeterminado (UICN, 1990).

La familia Bufonidae está representada por los géneros Bufo, con cinco especies, Nectophryne y Werneria, con una especie cada uno. La especie de Bufo más común en el Parque es B. camerunensis, y la más rara, incluida en el apéndice I del CITES, $B$. superciliaris, que se distribuye principalmente en las inmediaciones de los ríos Uoro y Laña. Werneria mertensiana suele encontrarse cerca de aguas corrientes. Por último, Nectophryne afra se conoce sólo de un ejemplar capturado en junio de 2002 durante la ya citada expedición del Museo Peabody. La presencia de esta especie en el Parque ya había sido supuesta por De la Riva (1994b). El ejemplar, colectado en las inmediaciones del Río Lobo, se hallaba en la boca de una culebra que lo acababa de capturar (Jorge de León, in litt.). Esta especie pone huevos en huecos de árboles o cual- 
quier otro pequeño recipiente natural (o incluso artificial) que se llene de agua, siendo la puesta cuidada por el macho (Scheel, 1970; Amiet, 1991).

La familia Hyperoliidae, con 16 especies, es la más diversa y la representan tres subfamilias: Kassininae (Phlyctimantis, 1 especie), Leptopelinae (Leptopelis, 7 especies, siendo el género de anfibio más diverso del Parque) e Hyperoliinae (cinco géneros, con un total de 8 especies). Incluye especies de amplio espectro ecológico, de hábitos fundamentalmente nocturnos, con especies distribuidas en cada uno de los tres tipos de hábitat del Parque, tanto en el suelo como en la vegetación arbórea, y asociadas o no a los cuerpos de agua; algunas especies de Afrixalus e Hyperolius son muy frecuentes en las zonas antrópicas.

La familia Pipidae está representada en el Parque por dos especies estrictamente acuáticas. Una de ellas, Hymenochirus boettgeri es aparentemente muy escasa, y sólo ha sido registrada en el río Michimi, afluente del Uoro, en las cercanías de Midyobo. Por el contrario, Xenopus fraseri, es relativamente abundante y ampliamente distribuida, hallándose tanto en aguas estancadas como en arroyos tranquilos. Se observaron grandes agrupaciones de larvas de esta especie en una charca temporal cerca de Moka el 29-10-93 y en una laguna temporal cerca de Bicurga (laguna Ncorotom) el 6-794; en ambos casos, los renacuajos formaban masas similares a cardúmenes de centenares de individuos cada uno.

La familia Ranidae, representada por las subfamilias Dicroglossinae (género Conraua, 2 especies), Petropedetinae (Dimorphognathus, Petropedetes, Phrynobatrachus, Phrynodon, con 7 especies en total), Ptychadeninae (Ptychadaena, 1 especie) y Raninae (Amnirana, 3 especies), constituye la segunda familia en importancia en cuanto al número de especies. Incluye la que tal vez sea la especie más abundante de la anurofauna del Parque, Phrynobatrachus auritus. En su conjunto, dentro de esta familia hay especies de hábitos diurnos y/o nocturnos cubriendo todos las hábitats y microhábitas considerados, algunas veces estrechamente ligadas al medio acuático (Conraua) y otras asociadas a las praderas y lajas de roca más elevadas del Parque (Petropedetes parkeri) (De la Riva, 1994b). Mención especial requiere Conraua goliath (Fig. 9), que, por su enorme tamaño, constituye una fuente de alimentación para los humanos $y$, por tanto, se encuentra en algún grado amenazada. En el PNMA se encuentra a lo largo de los ríos Laña, Uoro y tramos inferiores de los principales afluentes de éstos: ríos Mongó, Envoguyi, Michimi

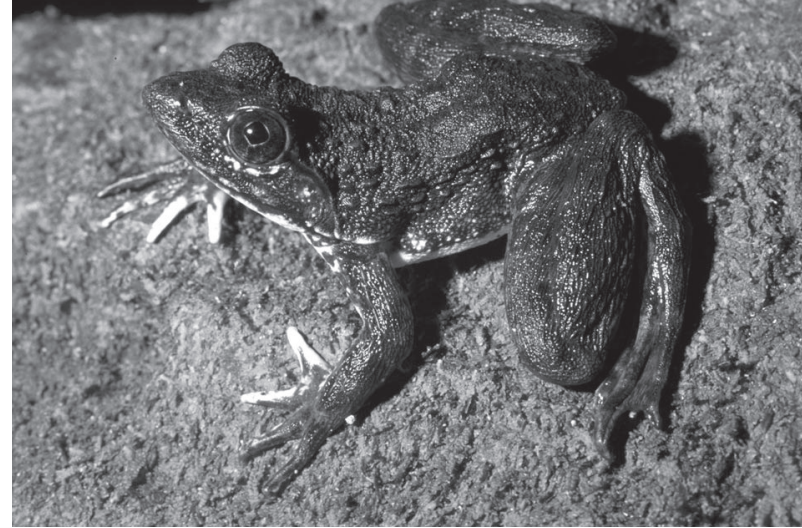

Fig. 9.- Conraua goliath.

y Bong (Uoro) y Bogo, Bilene, Menseng y Bibas (Laña). De la Riva (1994b) la señala para los rápidos Asia, Cong y Cascada Tutubo del río Uoro. Sabater Pi (1962, 1967 y 1985) indica su presencia en los rápidos de Sendje y Nguadung (Bajo Uoro) y cascadas del Laña cerca de la confluencia con el Uoro. En un censo nocturno realizado en el río Uoro el día 8-8-94 aguas abajo de Midyobo en un tramo entre los $01^{\circ} 44^{\prime} 09^{\prime \prime} \mathrm{N}-10^{\circ} 09^{\prime} 31^{\prime \prime} \mathrm{E}$, con sólo tres zonas de rápidos, se observaron siete individuos ( 2 juveniles y 5 adultos) en la margen izquierda y nueve individuos ( 7 juveniles y 2 adultos) en la margen derecha. De la Riva (1994b) señala ocho individuos juveniles en un tramo de $70 \mathrm{~m}$ del río Laña. Probablemente sea una especie más abundante en el Laña que en el Uoro, donde está sometida a mayor persecución por el hombre. De acuerdo a la UICN (1990), el estatus poblacional de esta especie en Río Muni permanece indeterminado. El PNMA representa aparentemente el límite sur de su distribución.

Por último, la familia Rhacophoridae está representada por una sola especie (Chiromantis rufescens), poco común en el Parque. Habita en la vegetación arbórea del bosque primario y secundario y ocasionalmente en la vegetación de mediana altura asociada a aguas estancadas, en donde la especie construye sus nidos.

\section{Discusión}

Como mencionamos en la introducción, la fauna de la región continental de Guinea Ecuatorial es aún menos conocida que la de Bioko, y los anfibios 


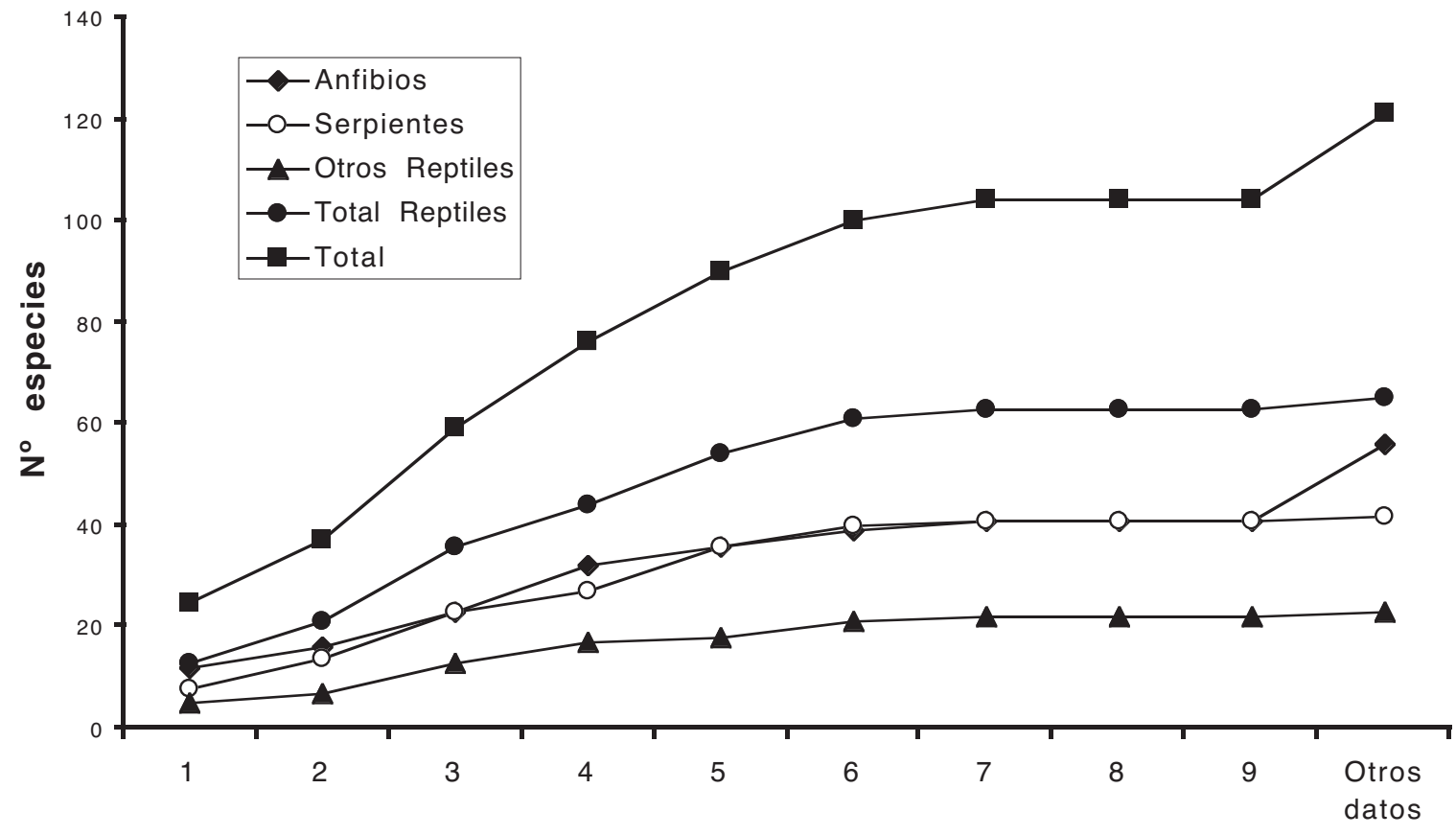

Meses

Fig. 10.- Curvas temporales de acumulación de especies encontradas durante nueve meses correspondientes al período mayo de 1994 - enero de 1995. En la parte derecha de la gráfica se alcanza el número total de especies conocidas incluyendo el muestreo de 1993 y otros datos disponibles.

Fig. 10.- Effective sampling success of the herpetofauna during the nine months corresponding to the period may 1994 - january 1995. At the right side of the figure, the total number of species is reached including the sampling period of 1993 and other data.

y reptiles constituyen el conjunto de vertebrados menos estudiado. La exhaustiva recopilación bibliográfica de Pérez del Val (2001) recoge sólo 19 referencias sobre anfibios y 15 de reptiles para Río Muni en todo el período 1831-2000, habiendo aparecido la primera referencia herpetológica en 1882. Por eso, no es de extrañar que aunque la presencia de muchas especies de amplia distribución en la región centro-africana fuese esperable en Río Muni, jamás se hayan publicado datos concretos al respecto, y cualquier estudio arroje datos interesantes y novedades. Por ejemplo, de la lista de 49 especies de anfibios citadas por De la Riva (1994b) para Monte Alén, 24 nunca se habían registrado en Río Muni con anterioridad, y el presente trabajo todavía añade a dicha lista otros cuatro géneros y siete especies para el Parque: G. seraphini, H. squalostoma, $N$. afra, C. escalerae, Schoutedenella sp., $H$. boettgeri y $L$. notatus, siendo cuatro de ellas además citadas por primera vez para Río Muni (Tabla 2). Cuatro de esas siete especies están entre las 16 especies de anfibios que De la Riva (1994b) comentó que podría esperarse encontrar en futuras prospecciones; las otras tres no eran esperadas. Estas cifras indican cuánto puede faltar aún por descubrirse y hacen prever que los anfibios del área podrían estar representados seguramente por unas 70 especies.

En cuanto a los reptiles, de las 65 especies registradas en el presente trabajo para Monte Alén, 21 constituyen primeros registros para Río Muni. Con seguridad, faltan bastantes especies de reptiles por encontrar en el Parque. Por ejemplo, sólo entre las serpientes, Chippaux (1999) incluye Río Muni en el área probable de distribución de 41 especies que hasta ahora no han sido encontradas en Monte Alén.

Monte Alén está, con seguridad, entre las zonas con mayor biodiversidad de Africa Central, pero aún estamos lejos de conocer completamente su herpetofauna. Las curvas de acumulación de especies encontradas durante el estudio de 1994-95 se empezaron a estabilizar aproximadamente al sépti- 
mo mes para todos los grupos estudiados (Fig. 10). Sin embargo, si a esto se suman los resultados del trabajo realizado en 1993, se observa un notable incremento, especialmente en la cifra correspondiente a los anfibios, por haber estado el trabajo de campo de ese año más sesgado hacia este grupo. A ello hay que añadir que durante la Expedición del Museo Peabody, orientada fundamentalmente hacia la ornitología, se encontraron al menos otras dos especies (un reptil y un anfibio) no registradas previamente. Estos datos ponen de manifiesto que futuras prospecciones, llevadas a cabo por diferentes investigadores, en distintas circunstancias y con distintas aproximaciones, con seguridad seguirán aportando nuevas especies para el Parque. Basándonos en todas la consideraciones anteriores, a título solamente aproximativo, podemos estimar que seguramente conocemos un $70-80 \%$ de la herpetofauna de Monte Alén, por lo que la cifra real de anfibios y reptiles que habitan el Parque bien podría estar en torno a 150 especies. Si futuras prospecciones confirman esta estima, la alta diversidad herpetofaunística de Monte Alén será comparable en el contexto de Africa central sólo con la de las faunas de algunas zonas de Camerún, como la región de Dja (149 especies; LeBreton, 1999), y Korup (172 especies; Lawson, 1993).

Todas estas cifras simplemente indican la imperiosa necesidad de continuar el inventario faunístico de Guinea Ecuatorial para así generar la información básica fundamental con la que han de contar las futuras políticas de conservación de los recursos naturales del país.

\section{AGRADECIMIENTOS}

A Luis Arranz, Director del Proyecto ECOFAC (Ecosistemas Forestales de Africa Central - Componente Guinea Ecuatorial), por su hospitalidad y ayuda durante nuestra estancia en Guinea. A la Asociación Amigos del Coto de Doñana por el apoyo logístico a este estudio. Ivan Ineich (Museo de Historia Natural de París - MNHN) confirmó la identificación o identificó varias de las especies de reptiles, Roger Barbour confirmó la identificación de las tortugas y Annemarie Ohler identificó las especies de anfibios adicionales al trabajo de De la Riva (1994), que contó entonces con la inestimable ayuda de J.-L. Amiet. Durante nuestra visita a París (MNHN) A. Dubois facilitó la consulta de la Colección de Herpetología. A Kristof Ziskowski y Jorge de León les agradecemos los datos sobre Nectophryne afra y, junto con W. Böhme, la información sobre la especie indeterminada de camaleón. Agradecemos a las autoridades de Guinea Ecuatorial pertenecientes al Ministerio de Agricultura, Pesca y Alimentación y a los representantes de AGRECO, en especial a F. Feys y C. Aveling, el apoyo institucional prestado. Por último, pero no menos importante, un agradecimiento muy especial a todos los pobladores de Monte Alen, Moka, el equipo de ecoguardas y los trabajadores de ECOFAC, sin los cuales no hubiera sido posible la realización de este trabajo. Esta investigación está enmarcada dentro del Programa ECOFAC, financiada por la C. C. E. (DG VIII) y dirigida por AGRECO - CTFT - Asociación Amigos del Coto de Doñana, y los costos de su publicación han sido parcialmente sufragados por el proyecto REN2001-1046 (Ministerio de Ciencia y Tecnología, España; investigador principal: Ignacio De la Riva).

\section{Referencias}

Amiet, J.-L., 1991. Images d'Amphibiens camerounais. III. Le comportement de garde des oeufs. Alytes, 9(1): 15-22.

Angel, F., 1933. Les Serpentes $d$ L'Afrique Occidental Francaise. Larose Editeurs. 246 pp.

Blanc, C. P. \& Frétey, T., 2000. Biogéographie des amphibiens d'Afrique centrale et d'Angola. Biogeographica, 76(3): 107-118.

Blanc, C. P. \& Frétey, T., 2002. Analyse zoogéographique du peuplement reptilien amphibiens de l'Afrique centrale et d'Angola. Biogeographica, 78(2): 49-75.

Boulenger, G. A., 1887. Catalogue of the Lizards in the British Museum (Natural History). Vol. III; Lacertidae, Gerrhosauridae, Scincidae, Anelytropidae, Dibamidae, Chamaeleonidae, 1-12. British Museum. London. 575 pp.

Boulenger, G. A., 1899. Descriptions of new batrachians in the collection of the British Museum (Natural History). Annals and Magazine of Natural History, 7(3): 273-277.

Boulenger, G. A., 1900. A list of the batrachians and the reptiles of the Gaboon (French Congo), with descriptions of new genera and species. Proceedings of the Zoological Society of London, 1900: 433-456.

Boulenger, G. A., 1903. Batraciens de la Guinée Espagnole. Memorias de la Sociedad Española de Historia Natural, 1: 61-64.

Boulenger, G. A., 1905. Reptiles de la Guinée Espagnole. Memorias de la Sociedad Española de Historia Natural, 1(8): 183-186.

Boulenger, G. A., 1906. Descriptions of new batrachians discovered by Mr. G. L. Bates in South Cameroon. Annals and Magazine of Natural History, 7(17): 317-323.

CARCASSON, R. H., 1964. A preliminary survey of the zoogeography of African butterflies. East African Wildlife Journal, 2: 122-157.

Castroviejo, J., Juste, J. \& Castelo, R., 1986. Proyecto Investigación y Conservación de la Naturaleza en Guinea Ecuatorial. Ministerio de Asuntos Exteriores. Madrid. 29 pp.

Chippaux, J.-P., 1999. Les serpents d'Afrique occidentale et centrale. Faune et Flora Tropicales 35. IRD, Paris. 278 pp. 
DE LA RIVA, I., 1994a. Informe sobre la diversidad faunistica del Parque Nacional de Monte Alen, Guinea Ecuatorial. Informe Técnico AGRECO - C.T.F.T. ASOCIACION AMIGOS DEL COTO DE DOÑANA. $51 \mathrm{pp}$.

DE LA RIVA, I., 1994b. Anfibios anuros del Parque Nacional de Monte Alen, Río Muni, Guinea Ecuatorial. Revista Española de Herpetología, 8: 123-139.

FA, J. E., 1991. Conservación de los Ecosistemas Forestales de Guinea Ecuatorial. UICN. Gland and Cambridge. $221 \mathrm{pp}$.

Frétey, T. \& Blanc, C. P., 2000. Les amphibiens d'Afrique centrale. Liste faunistique. ADIE. Libreville. 39 pp.

GuINEA, E., 1946. Ensayo Geobotánico de la Guinea Continental Española. Instituto de Estudios Africanos, CSIC. Madrid. 389 pp.

Hamilton, A., 1976. The significance of patterns of distribution shown by forest plants and animals in tropical Africa for the reconstruction of Upper Pleistocene palaeoenvironments: a review. Palaeocology of Africa, 9: 63-97.

Hughes, B., 1983. African snake faunas. Bonner Zoologische Beiträge, 34(1/3): 311-356.

Juste, J., 1993. Principales Ecosistemas Terrestres y Usos Tradicionales y Comerciales de sus Recursos Naturales. Proyecto de Conservación y Manejo de los Recursos Naturales. Guinea Ecuatorial. Informe Técnico. FORINDECO. 82 pp.

Juste, J. \& CANTERO, A., 1991. Informe Nacional sobre Medio Ambiente y Desarrollo. Guinea Ecuatorial. Proyecto PNUD / FAO. EGQ / 87 / 003. UNCED. 149 pp.

KeAY, R. W., 1959. Vegetation Map of Africa: South of the Tropic of Cancer. Oxford University Press \& UNESCO. London. 24 pp.

KNOEPFFler, L. F., 1965. Faune du Gabon (Amphibiens et Reptiles) I: Ophidiens d l'Ogooué-Ivindo et du Woleu N'tem. Biologia Gabonica, 2(1): 3-23.

KnOEPFFler, L. F., 1968. Clef de détermination des serpents actuallment connus du Gabon et des contrées limitrophes (Moyen-Congo, Río Muni, Cabinda et Sud Cameroun forestier). Biologia Gabonica, 4(2): 183-194.

KnoepfFler, L. F., Faune du Gabon (Amphibiens et Reptiles) II: Crocodiles, cheloniens et sauriens d l'Ogooué-Ivindo et du Woleu N'tem. Vie et Milieu, 24(1): 111-128.

Lasso, C. A., 1995. Biodiversidad Animal del Parque Nacional de Monte Alén. República de Guinea Ecuatorial, Proyecto ECOFAC - Componente Guinea Ecuatorial. Informe Técnico AGRECO C.T.F.T. - ASOCIACION AMIGOS DEL COTO DE DOÑANA. $78 \mathrm{pp}$.
Lawson, D. P., 1993. The reptiles and amphibians of Korup National Park Project, Cameroon. Herpetological Natural History, 1(2): 27-90.

LeBreton, M., 1999. A working checklist of the herpetofauna of Cameroon. Netherlands Committee for IUCN. Amsterdam. 160 pp.

LeJOLY, J., 1994. Mise en place des transects en vue des inventaires de biodiversité á Monte Alen (Guinée Equatoriale). Proyecto ECOFAC. Informe técnico AGRECO - C.T.F.T. 90 pp.

LOVERIDGE, A., 1972. Revision of the african lizards of the family Gekkonidae. Bulletin of the Museum of Comparative Zoology, 98(1): 1-672.

Maley, J., 1987. Fragmentation de la forêt dense humide africaine et extension des biotopes montagnards au Quaternaire récent: nouvelles données polliniques et chronologiques. Implications paléoclimatiques et biogéographiques. Palaeocology of Africa, 18: 307-334.

Maley, J., 1991. The African rain forest vegetation and palaeoenvironments during late Quaternary. Climatic Change, 19: 79-98.

Meirte, D., 1992. Clés de détermination des serpents d'Afrique. Annals du Musee Royal de l'Afrique Centrale, Sciences Zoologiques, 267: 1-152.

Moreau, R. E., 1963. Vicissitudes of the African biomes in the Late Pleistocene. Proceedings of the Zoological Society of London, 141: 395-421.

Moreau, R. E., 1966. The bird faunas of Africa and its islands. Academic Press. New York. 424 pp.

Parmentier, I., 1999. La végétation des inselbergs du Rio Muni. Canopée, Bulletin sur l'environnement en Afrique Centrale, 14: 7-9.

Parmentier, I. \& Nguema, N., 2001. La vegetación de los inselbergs de Río Muni. In: C. Aedo, R. Morales, M. T. Tellería y M. Velayos (eds). Botánica y botánicos en Guinea Ecuatorial. Real Jardín BotánicoCSIC. Madrid: 201-230.

PÉREZ DEL VAL, J., 2001. Bibliografía de fauna de Guinea Ecuatorial (1831-2000). Manuales Técnicos de Museología 12, MNCN-CSIC. Madrid. $122 \mathrm{pp}$.

Perret, J. L., 1966. Les amphibiens du Cameroun. Zoologische Jahrbücher. Abteilung für Systematik, Ökologie und Geographie der Tiere, 8: 289-464.

Perret, J. L., 1973. Contribution à l'étude des "Panaspis" (Reptilia, Scincidae) d'Afrique occidentale avec la description de deux espèces nouvelles. Revue Suisse Zoologie, 80(2): 595-630.

Perret, J. L., 1975. La différenciation dans les genres Panaspis Cope (Reptilia, Scincidae). Bulletin de la Societé Neuchateloise des Sciences Naturelles, 98:5-16.

SABAter Pi, J., 1962. Aportación al estudio de la rana Goliath Conraua goliath (Boulenger). Zoo, 2: 30-33. 
SABATER PI, J., 1967. Notas sobre la ecología de la rana gigante de Río Muni. Zoo, 7: 24-25.

SABATER PI, J., 1984. Gorilas y Chimpancés del África Occidental. Fondo de Cultura Económica. México. $284 \mathrm{pp}$.

Sabater PI, J., 1985. Contribution to the biology of the giant frog (Conraua goliath, Boulenger). AmphibiaReptilia, 6(2): 143-153.

SCHEEL, J. J., 1970. Notes on the biology of the African tree-toad, Nectrophryne afra Buccholz \& Peters, 1875 (Bufonidae, Anura) from Fernando Poo. Revue de Zoologie et Botanique Africaine, 81(3/4): 225-236.

SCHMIDT, K. P., 1919. Contributions to the herpetology of the Belgian Congo based on the collection of the Museum Congo expedition, 1909-1915. Bulletin of the American Museum of Natural History, 39: 385-624.
UICN, 1990. Biodiversity in Sub-Saharan Africa and its Islands. Conservation Management and Sustainable Use. Occasional Papers of the UICN Species Survival Commission, 6: 1-242.

Villiers, A., 1963. Les serpents de l'Ouest Africain. Mémoires de l'Institute Française de l'Afrique Noire, II (Segunda Ed.): 1-190.
Recibido, el 23-VIII-2002

Aceptado, el 22-XI-2002

Publicado, el 31-XII-2002 